\title{
Exploring a different geometry for the solar tachocline in a kinematic solar dynamo model
}

\author{
G. A. Guerrero ${ }^{1} \dagger$, E. M. de Gouveia Dal Pino ${ }^{1}$ \\ ${ }^{1}$ Department of Astronomy, Insituto de Astronomia, Geofísica e Ciências Atmosféricas, \\ IAG-USP, Brazil \\ email: guerrero,dalpino@astro.iag.usp.br
}

\begin{abstract}
Kinematic solar dynamo models (KSDM) that use a solar like differential rotation profile usually fail to reproduce the latitudinal distribution of the toroidal magnetic fields. Usually, it is assumed that it is the larger radial shear present in the solar tachocline at higher latitudes that is the responsible for such results. We here consider variations in the shape and thickness of the tachocline and find that a better distribution of the toroidal fields is obtained when the thickness of the tachocline is $d_{1}=0.02 R_{\odot}$, a smaller value than that conventionally used.
\end{abstract}

Keywords. Solar magnetic fields, MHD, Solar dynamo, tachocline

\section{Introduction}

The large scale solar magnetic cycle (LSSMC) can be summarized by its six more important phenomena: aparition of pairs of sunspots of oposite magnetic polarity in a belt of $\pm 30^{\circ}$ around the solar equator (Spöerer law); invertion of polarities in a mean period of 11 years (Hale's law); equatorward migration of sunspots and poleward migration of the dipolar diffuse field; phase lag between the inversion of polarities of toroidal and poloidal fields (when the first one reaches its maximum the last one undergoes a flip); and a maximum strength of the sunspots around $10^{3} \mathrm{G}$ and dipolar field around tens of G. All these fiatures suggest that the amplification and support of the magnetic field obeys an hydromagnetic dynamo process with the transformation of a poloidal field into a toroidal field, followed by the regeneration of the poloidal field but with opposite polarity to the initial one, and so on.

Since helioseismic measurements have indicated the presence of a tachocline, i.e., an interface between a uniform and a differential rotation layer, it has been a common believe that the dynamo action takes place in this thin layer with substantial radial and latitudinal shear. Within the tachocline, a poloidal magnetic field is stretched by the solar rotation, such that a belt of strong toroidal magnetic field is formed around the solar equator (the $\Omega$ effect). Theoretical arguments suggest that this field is not homogeneous but mainly concentred in magnetic flux tubes surrounded for less magnetized plasma. In the Babcock-Leighton scenario, the strong magnetic pressure can make these flux tubes rise to the surface by the action of magnetic buoyancy to form bipolar active magnetic regions (BMR's) in the surface. There, the poloidal field is possibly regenerated by the rise, twist and later decay of the BMR's. Meridional circulation plays an important role in the decay and advection of this poloidal field, and seems to be the most important parameter for setting the period of the cycle (Dikpati \& Charbonneau (1999)).

$\dagger$ Present address: IAG-USP, Rua do Matao, 1226, Sao Paulo, SP, Brazil 
Most of the models that consider a differential rotation profile derived from helioseismic inversions present an interesting problem, the radial shear, $\partial \Omega / \partial r$ (where $\Omega$ is the angular velocity and $r$ is the radial distance), is larger at higher latitudes than at lower ones so that a strong toroidal field is expected to be generated in regions closer to the poles and therefore sunspots should appear at higher latitudes, contradicting the observations (Spörer's Law). Apparently this was an unavoidable problem and no change in the parameters could solve it (Dikpati \& Charbonneau (1999), Kuker et al. (2001)), until Nandy \& Choudhuri (2002) proposed a new possible scenario in order to reduce the amplitude of the toroidal field at higher latitudes. They suggested that the meridional flow could penetrate below the tachocline, in the radiative zone. Thus the toroidal magnetic field would be stored in a more stable layer and advected by meridional circulation to lower latitudes from where it could then emerge to the tachocline and the convective zones and become buoyantly. This model (see also Chatterjee, Nandy \& Choudhuri (2004)) results a magnetic field distribution which is in qualitative agreement with the observations of the sunspots distribution, though it may affect the mixture of the elements and the observed abundances, and also the angular momentum transfer, but these specific potential problems will not be addressed in the present work (see, e.g., Guerrero \& Muñoz (2004).

In a recent work, Guerrero \& Muñoz (2004) have developed a CDSD model using a solar meridional circulation similar to that of Nandy \& Choudhuri (2002), but employed a source poloidal term used by Dikpati \& Charbonneau (1999). They have been able to reproduce the main figures of the LSSMC except the confinament of the toroidal field in lower latitudes, even using a deep meridional flow, showing that different solutions may arise to this problem which is highly model dependent.

In the present work, we consider a new approach to this problem by taking into account early helioseismic results of Charbonneau et al. (1999) that indicate that the tachocline could have a prolate shape. A numerical experiment of Dikpati \& Gilman (2001) have suggested that a strong magnetic stress with $B \sim 10^{5} \mathrm{G}$ can pile the matter into the poles generating a denser and thicker tachocline at higher latitudes. A thicker polar tachocline would imply a lower radial shear in that region and therefore, a smaller toroidal magnetic field could be expected.

In section $\S 2$, we present the mathematical formalism of our model. The details of the numerical tool employed can be found in Guerrero \& Muñoz (2004). The assumed profiles to construct the model and a previous solution are also shown. In section $\S 3$, we describe the main results of our model for different shapes and thicknesses of the tachocline, and draw our conclusions.

\section{Mathematics}

The MHD induction equation that governs the dynamo evolution can be divided in its poloidal $\left(\mathbf{B}_{\mathbf{p}}=\nabla \times A\right)$ and toroidal $\left(B_{\phi}=B\right)$ components:

$$
\begin{gathered}
\frac{\partial A}{\partial t}+\frac{1}{s}(\mathbf{u} \cdot \nabla)(s A)=\eta\left(\nabla^{2}-\frac{1}{s^{2}}\right) A+S_{1}(r, \theta, B) B \\
\frac{\partial B}{\partial t}+\frac{1}{r}\left[\frac{\partial}{\partial r}\left(r u_{r} B\right)+\frac{\partial}{\partial \theta}\left(u_{\theta} B\right)\right]=\left(\mathbf{B}_{\mathbf{p}} \cdot \nabla\right) \Omega-\nabla \eta \times \nabla \times B+\eta\left(\nabla^{2}-\frac{1}{s^{2}}\right) B
\end{gathered}
$$


Table 1. Value of parameters for a reference solution

\begin{tabular}{lcc}
\hline PARAMETER & VALUE & DESCRIPTION \\
\hline$\Omega_{E q}$ & $2 \pi \times 460.7 \mathrm{nHz}$ & equatorial angular velocity \\
$R_{c}$ & $0.7 R_{\odot}$ & location of the tachocline \\
$d_{1}$ & $0.05 R_{\odot}$ & thickness of the solar tachocline \\
\hline$U_{0}$ & $25 \mathrm{~m} \mathrm{~s}^{-1}$ & meridional velocity at middle latitudes \\
$R_{p}$ & $0.69 R_{\odot}$ & deep of penetration of mer. flow \\
\hline$\eta_{r}$ & $2.2 \times 10^{8} \mathrm{~m}^{2} \mathrm{~s}^{-1}$ & radiative core diffusivity \\
$\eta_{c}$ & $8.8 \times 10^{1} 0 \mathrm{~m}^{2} \mathrm{~s}^{-1}$ & convective zone diffusivity \\
$\eta_{s}$ & $1.0 \times 10^{1} 2 \mathrm{~m}^{2} \mathrm{~s}^{-1}$ & superficial diffusivity \\
\hline$\alpha_{0}$ & $100 \mathrm{~m} \mathrm{~s}^{-1}$ & amplitude of $\alpha$ effect \\
\hline
\end{tabular}

where $\Omega$ is the angular velocity, $\mathbf{u}=\mathbf{u}_{\mathbf{r}}+\mathbf{u}_{\theta}$ is the velocity in the meridional plane, $s=r \sin \theta$ and $\eta$ is the magnetic diffusivity coefficient. In the right hand side of eq. (2.1), $S_{1}\left(r, \theta, B_{\phi}\right)$ is the source term of the poloidal field that contains the Babcock-Leighton concept of emergence of magnetic flux tubes and generates a poloidal field until a saturation value $B_{0}=10^{5} \mathrm{G}$ (the $\alpha$ term). Besides these, we may distinguish two more ingredients in these equations: the differential rotation and the meridional circulation. While helioseismology mesurements give an accurate profile of the angular velocity, the movement at the meridional plane is known only in the outer layers. For the differential rotation, we use the analytical profile introduced by Dikpati \& Charbonneau (1999) and a single convection cell for the quadrant meridional flow (see eq. 8 of Nandy \& Choudhuri (2001)). We use three different regimes for the magnetic diffusivity: with molecular diffution values for the radiative and the the convective layers and an observed supergranular diffusion value for a thin layer close to the surface (see Fig. 5 of Dikpati et al. (2004)). Finally, we use a non-local and non-linear $\alpha$ term that operates close to the surface and at the latitudes where the sunspots appear (Eq. 11 and Figs. 7 of Dikpati et al. (2004)).

Employing the profiles above and the parameter values shown in Table 1 we have built the butterfly diagram depicted in Fig. 1a. We notice, however, that although it can reproduce qualitatively the magnetic field distribution and periodicity of the sunspots, a strong $\left(\leqslant 4.5 \times 10^{4} \mathrm{G}\right)$ toroidal magnetic field appears close to the poles.

\section{A prolate or a thinner tachocline?}

Considering a tachocline with prolate angular velocity profiles (with $d_{1}=0.07 R_{\odot}$ in the poles and $d_{1}=0.02 R_{\odot}$ in the equator), we have obtained the butterfly diagram of Fig. 1b. We note that the latitudinal distribution of toroidal fields remains unaltered. However, when an oblate geometry is used instead (Fig. 1c), we find that the toroidal fields are amplified to their maximum value only below the $60^{\circ}$ latitudes. In view of this result, we assumed again a spherical tachocline but with a thinner thickness, obtaining in this case a butterfly diagram that is in good agreement with the observations (Fig. 1d). These results suggest that either the real thickness of the tachocline is smaller than the usually assumed value or that the dynamo process does not involve the entire tachocline but only a fraction of it. A more detailed discussion of these results is presented elsewhere (Guerrero \& de Gouveia Dal Pino, 2006, in prep.).

\section{Acknowledgements}

This project has been partially supported by CNPq grants. 

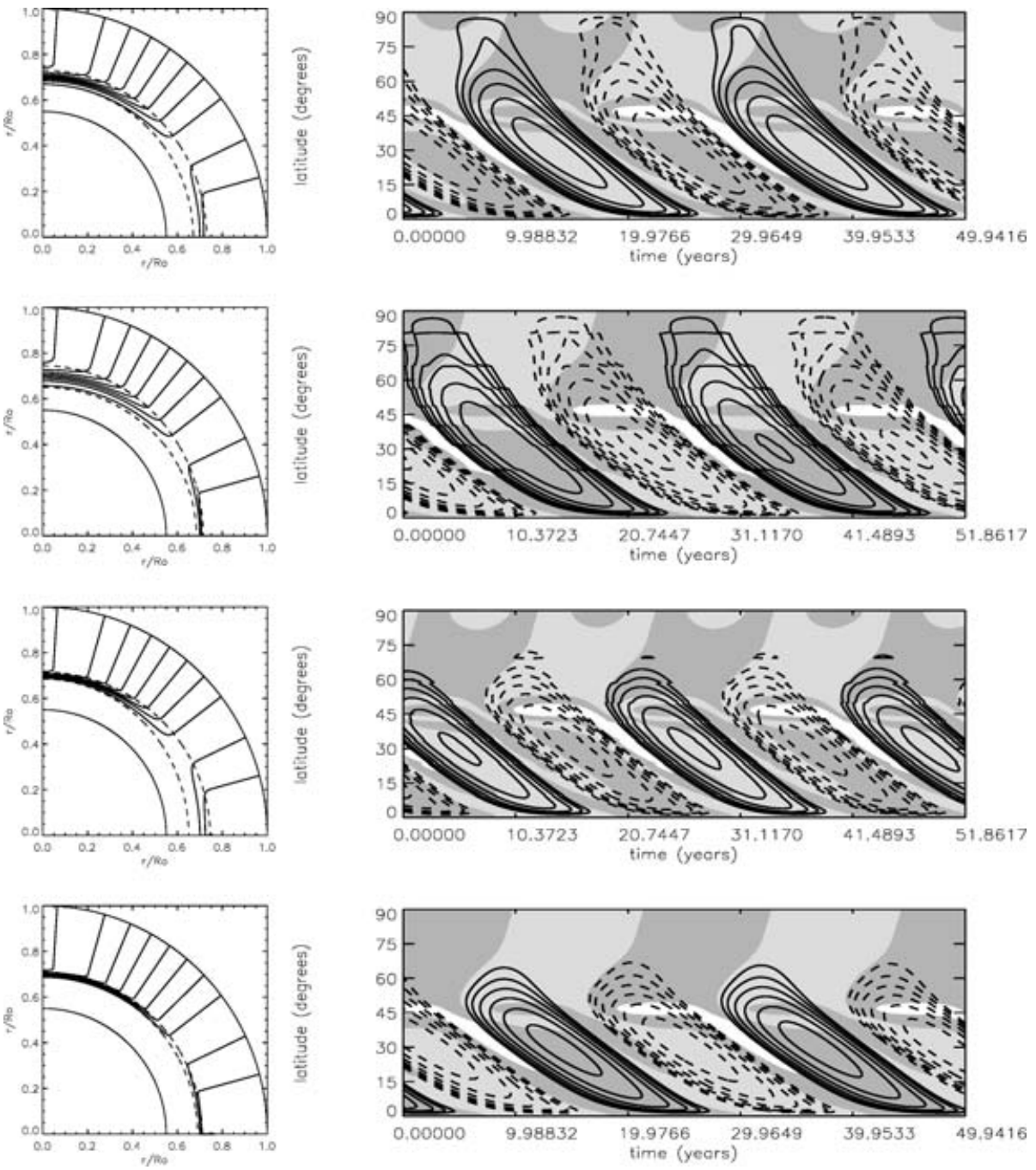

Figure 1. Angular velocity profiles and time-latitude butterfly diagrams for: a) a spherical tachocline (with the initial conditions described in Table 1); b) a prolate shape tachocline; c) an oblate shape tachocline; and d) a thinner spherical tachocline (see the text for details). Continous/dashed lines represent positive/negative values of the magnetic field strength in the top of the tachocline $\left(r=R_{t}+d_{1} / 2\right)$. The lines are log spaced and cover the interval between $4.5 \times 10^{4}$ and $5.5 \times 10^{5} \mathrm{G}$. The grey shaded background represents the surface radial fields.

\section{References}

Charbonneau, P., Chistensen-Dalsgaard, J., Hening, R., Schou, J., Thompson, M. J., Tomczy, S. 1999, ApJ 527, 445

Kuker, M., Rüdiger, G., Schultz, M. 2001 A $\&$ \& 374, 301

Dikpati, M., Charbonneau, P. 1999 ApJ 518, 508

Chatterjee, P., Nandy, D., Choudhuri, R. A. 2004, A\&\&A 427, 1019

Nandy, D., Charbonneau, P. 2002, Science 306, 1671

Guerrero, G., Muoz, J. D. 2004, MNRAS 350, 317

Dikpati, M., de Toma, J., Gilman, P. 2004 ApJ 601, 1136

Dikpati, M., Gilman, P. 2004 ApJ 552, 348

Nandy, D., Choudhuri, R. A. 2001 ApJ 551, 576 